\title{
Bidirectional ephrinB2-EphB4 signaling regulates the osteogenic differentiation of canine periodontal ligament stem cells
}

\author{
SHAOYUE ZHU ${ }^{1,2^{*}}$, ZONGXIANG LIU ${ }^{3 *}$, CHANGYONG YUAN $^{3}$, YIFAN LIN $^{1}$, YANQI YANG ${ }^{1}$, \\ HAIMING WANG ${ }^{1}$, CHENGFEI ZHANG $^{4}$, PENGLAI WANG ${ }^{5}$ and MIN GU ${ }^{1}$ \\ ${ }^{1}$ Discipline of Orthodontics and Paediatric Dentistry, Faculty of Dentistry, The University of Hong Kong, \\ Hong Kong, SAR 999077; ${ }^{2}$ Department of Orthodontics and ${ }^{3}$ Discipline of Oral and Maxillofacial Surgery, \\ Affiliated Stomatological Hospital of Xuzhou Medical University, Xuzhou, Jiangsu 221000; ${ }^{4}$ Department of Endodontology, \\ Faculty of Dentistry, The University of Hong Kong, Hong Kong, SAR 999077; ${ }^{5}$ Dental Implant Center, \\ Affiliated Stomatological Hospital of Xuzhou Medical University, Xuzhou, Jiangsu 221000, P.R. China
}

Received June 11,2019; Accepted December 23, 2019

DOI: $10.3892 / \mathrm{ijmm} .2020 .4473$

\begin{abstract}
The aim of the present study was to evaluate the effect of ephrinB2 gene-transfected canine periodontal ligament stem cells (cPDLSCs) on the regulation of osteogenic differentiation. cPDLSCs were transfected with a transgenic null-control green fluorescent protein (GFP) vector (termed Vector-cPDLSCs) or with NFNB2 GFP-Blasticidin (termed EfnB2-cPDLSCs). Subsequently, the osteogenic differentiation of Vector-cPDLSCs and EfnB2-cPDLSCs was assessed by reverse transcription-quantitative polymerase chain reaction (RT-qPCR), alkaline phosphatase (ALP) assay and Alizarin Red S staining. The migratory abilities of cPDLSCs, Vector-cPDLSCs and EfnB2-cPDLSCs were also assessed. Following osteogenic induction of Vector-cPDLSCs and EfnB2-cPDLSCs, the protein expression levels of collagen I, Runt-related transcription factor 2, osteocalcin, ephrin type-B receptor 4 (EphB4), phospho-EphB4, ephrinB2 and phospho-ephrinB2 were analyzed by western blot assays. Following gene transfection, the RT-qPCR and western blotting results revealed that the mRNA and protein expression levels of ephrinB2, respectively, were significantly increased in EfnB2-cPDLSCs compared with that in Vector-cPDLSCs
\end{abstract}

Correspondence to: Professor Penglai Wang, Dental Implant Center, Affiliated Stomatological Hospital of Xuzhou Medical University, 130 Huaihaixi Road, Xuzhou, Jiangsu 221000, P.R. China E-mail:wp10771@163.com

Dr Min Gu, Discipline of Orthodontics and Paediatric Dentistry, Faculty of Dentistry, The University of Hong Kong, Hong Kong, 34 Hospital Road, Hong Kong, SAR 999077, P.R. China

E-mail:drgumin@hku.hk

${ }^{*}$ Contributed equally

Key words: EphB4/ephrinB2 signaling, osteogenic differentiation, canine periodontal ligament stem cells
$(\mathrm{P}<0.05)$. ALP and Alizarin Red S staining assays revealed increased ALP activity and mineralization nodules, respectively, in EfnB2-cPDLSCs. Cell proliferation and migration assays revealed that EfnB2-cPDLSCs exhibited enhanced proliferation and migration compared with Vector-cPDLSCs $(\mathrm{P}<0.05)$. In conclusion, the findings of the current study indicated that ephrinB2 gene-modified cPDLSCs exhibited enhanced osteogenic differentiation, with the ephrinB2 reverse signaling and EphB4 forward signaling pathways serving a key role in this process. Furthermore, ephrinB2 gene modification was observed to promote the migration and proliferation of cPDLSCs.

\section{Introduction}

Periodontitis can cause progressive destruction of dental support tissue, which is the primary risk factor for tooth loss in adults (1-3). Traditional non-surgical treatments, such as scaling, curettage and root surface leveling, can only remove plaque and reduce inflammation (4), while surgical treatments such as guided tissue regeneration and guided bone regeneration have limited regenerative effects (5). Technologies that can achieve periodontal tissue regeneration and functional recovery currently remain under investigation. In recent years, periodontal tissue engineering has developed rapidly, providing a new direction for research. Periodontal tissue engineering is a technique that uses tissue engineering to construct a complex composed of one or more elements (such as cells, scaffolds and signaling molecules) for implantation into the defect area to achieve functional tissue regeneration $(6,7)$. However, ongoing challenges for this technique include the identification of the best source of cells to construct such tissue-engineered complexes, as well as the selection of biological signaling molecules to stimulate the regenerative potential of implanted stem cells in order to enhance their therapeutic effects.

It has been reported that the ephrinB2/ephrin type-B receptor 4 (EphB4) signaling pathway serves an important role in bone remodeling processes. The reverse signaling pathway mediated by ephrinB2 inhibits the function of osteoclasts and 
bone resorption by decreasing c-Fos and NFATc1 activity (8). By contrast, the forward signaling pathway mediated by EphB4 enhances osteoblast differentiation and promotes bone formation by decreasing RhoA activity (9). In addition, ephrinB2-Fc can bind to EphB4 receptors on the surface of endothelial cells and promote the migration of vascular endothelial cells by activating the phosphatidylinositol 3-kinase signaling pathway to accelerate angiogenesis (10-12). Our previous experiments verified that ephrinB2 gene-transfected dental stem cells enhanced the osteogenic ability and promoted the angiogenesis of peripheral vascular endothelial cells (13), as well as promoted their angiogenesis in co-culture systems (14). In addition, we observed that the extracellular matrix of umbilical vein endothelial cells was able to promote the differentiation of odontogenic stem cells into endothelial cells (15). Therefore, it can be speculated that the ephrinB2 signaling molecule enhances cell migration, promotes odontogenic stem cell osteogenesis and enhances peripheral angiogenesis. As such, ephrinB2 is an ideal biological signaling molecule for osteogenic regeneration.

Previous studies reported that a multitude of mature stem cells can be derived from dental tissues, including periodontal ligament stem cells (PDLSCs), dental pulp stem cells (DPSCs), apical papilla and stem cells from human exfoliated deciduous teeth (SHEDs), all of which exhibit a potent capacity for differentiation into mesodermal lineages (16-18). Mesenchymal stem cells possessing stem-like features can produce osteo/odontogenic, adipogenic and neurogenic lineages. Notably, DPSCs and SHEDs have been successfully induced into endothelial cells both in vitro and in vivo (19-22), as have bone marrow mesenchymal stem cells and human adipose-derived stem cells $(23,24)$. Collectively, these results indicate that dental stem cells are a suitable candidate for tissue engineering.

While non-animal methods are widely used in biomedical research, they cannot replace all use of animals. Currently, the canine mandible defect model is widely used in preclinical studies (25-27). Notably, beagle dogs are recommended as potential animal models for medical testing due to their genetic, biological and behavioral characteristics that closely resemble those of humans; furthermore, numerous symptoms of human conditions can be replicated in dogs (28). Previous studies comparing canine and human dental mesenchymal stem cells have demonstrated that canine DPSCs were able to differentiate into odontoblast-like cells exhibiting osteogenic potential similar to human DPSCs (29). However, in vitro transfection of canine PDLSCs (cPDLSCs) with ephrinB2 and the use of transfected cells as seed cells for tissue engineering have seldom been investigated. In addition, how ephrinB2/EphB4 signaling may facilitate the osteogenic/odontogenic differentiation of cPDLSCs has not been previously investigated. Therefore, the main focus of the current study was to address these issues.

\section{Materials and methods}

Isolation, culture and identification of cells. cPDLSCs were isolated from 6-month-old beagle dogs. A total of 4 6-month old male beagle dogs (JC0853, $8.0 \mathrm{~kg}$; JC0857, $7.5 \mathrm{~kg}$; JC0889, $6.6 \mathrm{~kg}$; JC0899, $7.4 \mathrm{~kg}$ ) were obtained from the Experimental Animal Center of Xuzhou Medical University (Xuzhou,
China). The dogs were bred carefully and exclusively by professional breeders and housed in individual and clean cages at room temperature with a humidity of $60 \%$, and regular food and water were provided twice daily. Ethical approval for the present study was obtained from the Institutional Animal Care and Use Committee of Xuzhou Medical University (Xuzhou, China; approval no. 20161108). Briefly, the dogs were induced to initial anesthesia by intravenous injection of $6 \mathrm{mg} / \mathrm{kg}$ propofol and maintained by inhalation of isoflurane at $2 \%$, with the oxygen flow maintained at $3 \mathrm{l} / \mathrm{min}$ for $1 \mathrm{~min}$ and then adjusted to $0.4 \mathrm{l} / \mathrm{min}$. The upper and lower anterior teeth were extracted, using phosphate-buffered saline containing penicillin and streptomycin to wash and separate the periodontal ligament. Next, the periodontal ligament tissue was scraped from the teeth and shattered on a super-clean table using a mixture of $3 \mathrm{mg} / \mathrm{ml}$ collagenase I (Gibco; Thermo Fisher Scientific, Inc.) and $4 \mathrm{mg} / \mathrm{ml}$ neutral protease (Dispase; (Gibco; Thermo Fisher Scientific, Inc.) to digest and extract the cells. The cells were cultured in $\alpha$-minimum essential medium ( $\alpha$-MEM; Gibco; Thermo Fisher Scientific, Inc.) supplemented with $20 \%$ fetal bovine serum (FBS; Thermo Fisher Scientific, Inc.) in a $37^{\circ} \mathrm{C}, 5 \% \mathrm{CO}_{2}$ incubator. The limiting dilution method was adopted to separate cPDLSCs from total canine periodontal ligament cells and their morphology was monitored under an inverted microscope. Flow cytometric analysis for STRO-1 (cat. no. 14-6688-82; Thermo Fisher Scientific, Inc.), CD45 (cat. no. MCA2035S; Bio-Rad Laboratories, Inc.), CD73 (cat. no. 202122; Abcam), CD90 (cat. no. ab139364; Abcam) and CD105 (cat. no. ab156756; Abcam) antibodies (all 1:500) was employed to detect the phenotype of cells. Briefly, cells were incubated with primary antibodies for $2 \mathrm{~h}$ in dark at room temperature and then washed with PBS. This was followed by incubation with the following appropriate secondary antibodies: Goat anti-mouse IgG H\&L (Alexa Fluor ${ }^{\circledR} 488$ ) pre-adsorbed (1:2,000; cat. no. ab150117; Abcam) and goat anti-rabbit IgG H\&L (Alexa Fluor ${ }^{\circledR} 488$; 1:2,000; cat. no. ab150077; Abcam) for $30 \mathrm{~min}$ in the dark at room temperature. A BD FACSVerse ${ }^{\mathrm{TM}}$ flow cytometer (BD Biosciences) was used to detect cells and BD FACSuite software V1.0 (BD Biosciences) were used to detect and analyze flow cytometry data.

Furthermore, other appropriate methods were utilized, as described below, to assess multidirectional differentiation ability of the cells. For osteogenic differentiation, cPDLSCs were seeded at a density of $1 \times 10^{4} /$ well in 12 -well plates and cultured to $70 \%$ confluence. Subsequently, the osteogenic induction medium ( $\alpha$-MEM containing 10\% FBS, $50 \mu \mathrm{g} / \mathrm{ml}$ L-ascorbic acid phosphate, $10 \mathrm{mmol} / \mathrm{l} \beta$-glycerophosphate and $10 \mathrm{nmol} / \mathrm{l}$ dexamethasone) was added. Following 4 weeks of culture at $37^{\circ} \mathrm{C}$, cells were fixed with $4 \%$ paraformaldehyde for $30 \mathrm{~min}$ and stained with $0.2 \%$ Alizarin Red S solution for $10 \mathrm{~min}$ at room temperature for mineralized nodules. For adipogenic differentiation, cPDLSCs, seeded at a density of $1 \times 10^{4} /$ well, were cultured to $70 \%$ confluence and cultured with odipogenic induction medium ( $\alpha$-MEM containing $10 \%$ FBS, $1 \mu \mathrm{g} / \mathrm{ml}$ insulin, $1 \mu \mathrm{mol} / 1$ dexamethasone and $0.5 \mathrm{mmol} / 1$ 3 -isobutyl-1-methylxanthine) for 4 weeks at $37^{\circ} \mathrm{C}$. Finally, the lipid droplets were stained with $0.5 \%$ Oil Red $\mathrm{O}$ for $10 \mathrm{~min}$ at room temperature. For neurogenic differentiation, cPDLSCs seeded at a density of $1 \times 10^{4} /$ well in 12 -well plates were cultured in Neurobasal A medium (Gibco; Thermo Fisher Scientific, 
Inc.) supplemented with $20 \mathrm{ng} / \mathrm{ml}$ epidermal growth factor (PeproTech, Inc.) and $40 \mathrm{ng} / \mathrm{ml}$ basic fibroblast growth factor (bFGF; PeproTech, Inc.) for 4 weeks at $37^{\circ} \mathrm{C}$. $\beta$ III-tubulin was detected by immunofluorescence.

Immunofluorescence analysis. Following rinsing with PBS, cPDLSCs were grown on glass cover slides, fixed with $4 \%$ paraformaldehyde for $10 \mathrm{~min}$ at room temperature and blocked with $1 \%$ BSA for $30 \mathrm{~min}$ at room temperature. Slides were incubated with primary mouse anti- $\beta$ III-tubulin antibody (1:500; Abcam; cat. no. ab119100) at room temperature in a humidified chamber overnight. Following thorough rinsing with PBS, slides were then incubated with goat anti-mouse IgG $(\mathrm{H}+\mathrm{L})$ secondary antibody overnight. Subsequently, 4,6-diamidino-2-phenylindole was used to label cell nuclei. The cover slides were sealed with an aqueous-based mounting medium (Vector Laboratories). For immunofluorescence microscopy, an inverted Nikon fluorescence microscope (Nikon Corporation) with a digital camera (magnification, $\mathrm{x} 20$ ) and deconvolution software (Slidebook 4.0; Intelligent Imaging) were used.

Transfection and identification of ephrinB2 gene-transfected cPDLSCs. cPDLSCs were transfected with a null-control green fluorescent protein (GFP)-Blasticidin Vector (Vector-cPDLSCs) or NFNB2 GFP-Blasticidin Vector (EfnB2-cPDLSCs). Approximately 18-24 h before transfection, adherent second-generation cPDLSCs were transferred to a 24-well plate at a density of $1 \times 10^{5} /$ well. The density of lentivirus-transfected cells was $\sim 2 \times 10^{5} /$ well. The following day, the original medium was replaced with $2 \mathrm{ml}$ fresh medium containing $6 \mu \mathrm{g} / \mathrm{ml}$ polybrene, and $10 \mu \mathrm{g}$ of the virus suspension was added for incubation at $37^{\circ} \mathrm{C}$ for $4 \mathrm{~h}$. Next, $2 \mathrm{ml}$ fresh medium was added to dilute the polybrene, and the cells were cultured for a further $24 \mathrm{~h}$. Subsequently, the medium containing the virus was replaced with fresh medium, and cells were cultured for another $24 \mathrm{~h}$. After $24 \mathrm{~h}$ of transfection, GFP fluorescence was observed under a fluorescent-inverted phase-contrast microscope (Olympus Corporation). After 3-4 days of transfection, Blasticidin-specific antibiotics (Merck $\mathrm{KGaA}$ ) were added continuously with monitoring to ensure that the cells were subcultured normally. Following Blasticidin selection, $1 \times 10^{5}$ EfnB2-cPDLSCs and vector-PDLSCs were cultured in six-well plates at $37^{\circ} \mathrm{C}$ for 48 or $72 \mathrm{~h}$. Reverse transcription-quantitative polymerase chain reaction (RT-qPCR) and western blotting were used to respectively detect gene and protein expression levels of ephrinB2 in vector-cPDLSCs and EfnB2-cPDLSCs following transfection. Wild-type cPDLSCs were used as control. All experiments were performed in triplicate.

Cell proliferation assay. A Cell Counting Kit-8 (CCK-8; Sigma Aldrich; Merck KGaA) assay was used to assess cell proliferation. Briefly, cPDLSCs, EfnB2-cPDLSCs or Vector-cPDLSCs were seeded at a density of $1 \times 10^{4}$ cells per well into a 96-well plate overnight $\left(37^{\circ} \mathrm{C}, 5 \% \mathrm{CO}_{2}\right)$. Next, $10 \mu \mathrm{lCK}-8$ solution was added to each well, followed by incubation for $4 \mathrm{~h}\left(37^{\circ} \mathrm{C}\right.$, $5 \% \mathrm{CO}_{2}$ ). The absorbance of each well was read at $450 \mathrm{~nm}$ with a microplate reader on days $0,2,4,6$ and 8 to assess the cell numbers.
Cell migration assay. A Transwell cell migration assay was used to detect the cell migration as previously described (30). Briefly, Transwell filter inserts were placed in 24-well tissue culture plates (Boyden chambers; BD LabWare), and after $12 \mathrm{~h}$ of serum starvation, $5 \times 10^{4}$ cPDLSCs, EfnB2-cPDLSCs or Vector-cPDLSCs were cultured in the upper chamber with serum-free $\alpha$-MEM. $\alpha$-MEM supplemented with $15 \%$ fetal bovine serum was added to the lower chamber. At 6, 8, 10 and $12 \mathrm{~h}$, viable cells that had migrated to the lower membrane surface were fixed with $4 \%$ (w/v) paraformaldehyde for $30 \mathrm{~min}$ at room temperature and stained with $0.1 \%(\mathrm{w} / \mathrm{v})$ crystal violet for $10 \mathrm{~min}$ at room temperature. Images were captured using an inverted microscope (Olympus Corporation) and counted to quantify the migrated cells.

Detection of osteogenic and angiogenic abilities of cPDLSCs in vitro. Vector-cPDLSCs and EfnB2-cPDLSCs were separately cultured in osteogenic induction medium ( $\alpha$-MEM containing $10 \% \mathrm{FBS}, 50 \mu \mathrm{g} / \mathrm{ml} \mathrm{L}$-ascorbic acid phosphate, $10 \mathrm{mmol} / \mathrm{l} \beta$-glycerophosphate, and $10 \mathrm{nmol} / \mathrm{l}$ dexamethasone) for 7,14 or 21 days. The expression levels of osteogenesisand angiogenesis-associated proteins were then detected by western blotting, as described later in the text. Alkaline phosphatase (ALP) assay and Alizarin Red S staining were also performed. Briefly, for ALP assay, the BCIP/NBT ALP Color Development kit (Beyotime Institute of Biotechnology) was used on day 14 of induction, and the ALP activity was assessed by an ALP Detection kit (Jiancheng Bioengineering Institute) on days 14, following the manufacturer's protocol. For Alizarin Red S staining, the cultures were fixed with $4 \%$ paraformaldehyde for $30 \mathrm{~min}$ at room temperature after 3 weeks of incubation $\left(37^{\circ} \mathrm{C}, 5 \% \mathrm{CO}_{2}\right)$ and then stained with $0.2 \%$ Alizarin Red S (Sigma-Aldrich; Merck KGaA) solution for $10 \mathrm{~min}$ at room temperature for mineralized nodules. To quantify the calcium mineralization of EfnB2-cPDLSCs and Vector-cPDLSCs within each well, sodium dodecyl sulfate (SDS; $10 \% \mathrm{w} / \mathrm{v})$ solution was added, and the cells were incubated overnight at $37^{\circ} \mathrm{C}$. The absorbance of lysates at $405 \mathrm{~nm}$ was then read with a SpectraMAX $340^{\circledR}$ microplate reader (Molecular Devices, LLC).

In order to investigate the mechanism of ephrinB2 in regulating osteogenic differentiation, ephrinB2 was blocked with ephrinB2 small interfering RNA (EphrinB2-siRNA; Thermo Fisher Scientific, Inc.). Briefly, cPDLSCs were seeded on 6-well plates until $70 \%$ confluence was reached, and then $30 \mu$ l Opti-MEM along with $500 \mu 1$ Lipofectamine 2000 and RNAiMAX reagent (all from Thermo Fisher Scientific, Inc.) were added to the cells. Next, $3 \mu \mathrm{l}$ siRNA was diluted in $150 \mu \mathrm{l}$ Opti-MEM prior to mixing it with the diluted RNAiMAX and Lipofectamine at a ratio of 1:1. The siRNA-lipid complex was incubated for $5 \mathrm{~min}$ at room temperature. Subsequently, cPDLSCs were incubated with the siRNA-lipid complex for $24 \mathrm{~h}$ prior to the osteogenic induction experiments, and then ALP and Alizarin Red S staining assays were performed. FAM-labeled negative control (NC)-siRNA (CN2001; Biomics Biotechnologies Co., Ltd.) was used as the control in this experiment.

$R T$ - $q P C R$.EfnB2-PDLSCs and Vector-cPDLSCs were cultured for 7, 14 or 21 days in normal $\alpha$-MEM or osteogenic/odontogenic 
induction medium, and then total RNA was extracted with an RNeasy Plus Mini kit (Qiagen) and quantified using a NanoDrop2000 spectrophotometer (Thermo Fisher Scientific, Inc.). Subsequently, $1.0 \mu \mathrm{g}$ total RNA was reverse transcribed into cDNA in a total reaction mixture of $10 \mu \mathrm{l}$ using Super-Script VILO Master mix (Invitrogen; Thermo Fisher Scientific, Inc.). qPCR was then conducted using an ABI Prism 7000 Sequence Detection system with SYBR-Green reagent (both from Applied Biosystems; Thermo Fisher Scientific, Inc.). The reaction program used for $\mathrm{qPCR}$ was as follows: $95^{\circ} \mathrm{C}$ for $10 \mathrm{~min}, 40$ cycles of $95^{\circ} \mathrm{C}$ for $15 \mathrm{sec}$ and $60^{\circ} \mathrm{C}$ for $1 \mathrm{~min}$, followed by melt curve analysis at $95^{\circ} \mathrm{C}$ for $15 \mathrm{sec}, 60^{\circ} \mathrm{C}$ for $1 \mathrm{~min}, 95^{\circ} \mathrm{C}$ for $15 \mathrm{sec}$ and $60^{\circ} \mathrm{C}$ for $15 \mathrm{sec}$. The relative expression values of the target genes were determined using the $2^{-\Delta \Delta \mathrm{Cq}}$ method (31) by normalization with the housekeeping gene GAPDH as an internal control. The primer sequences are listed in Table I. Standards and samples were run in triplicate.

Western blot analysis. Vector-cPDLSCs and EfnB2-cPDLSCs were seeded at a density of $1 \times 10^{5}$ cells per well in 6 -well plates. When the cells reached $70 \%$ confluence, $2 \mu \mathrm{g} / \mathrm{ml}$ erythropoietin-producing human hepatocellular receptors B4 fragment (EphB4-FC, R\&D Systems) was added to activate ephrinB2 ligand. The cells were stimulated with EphB4-FC for $0,5,10,20,30$ and $60 \mathrm{~min}$ or induced with osteogenic medium for $0,12,24,48$ and $72 \mathrm{~h}$, and then M-PER ${ }^{\mathrm{TM}}$ Protein Extraction buffer containing $1 \mathrm{X}$ protease inhibitor cocktail (Thermo Fisher Scientific, Inc.) was used to extract the total protein. A BCA kit (Thermo Fisher Scientific, Inc.) was used to quantify the protein concentrations. Next, protein samples were separated by 7.5 or $12 \%$ SDS-polyacrylamide gel electrophoresis and transferred onto an ImmunBlot PVDF membrane (EMD Millipore). Membranes were blocked with 5\% bovine serum albumin in Tris-phosphate buffer containing $0.05 \%$ Tween-20 (TBS-T) for $1 \mathrm{~h}$ at room temperature, and further incubated overnight at $4^{\circ} \mathrm{C}$ with primary antibodies specific for ephrinB2 (1:1,000; ab131536; Abcam), phospho-ephrinB2 (1:1,000; ab119323; Abcam), EphB4 (1:1,000; sc-5536; Santa Cruz Biotechnology), phospho-EphB4 (1:500; 12720; Signalway Antibody), collagen 1 (1:500; COL1; ab6308; Abcam), Runt-related transcription factor 2 (1:1,000; Runx2; ab76956; Abcam), osteocalcin (1:1,000; OCN; 33-5400; Thermo Fisher Scientific, Inc.) and $\beta$-actin (1:2,000; sc-47778; Santa Cruz Biotechnology). Following three washes with TBS-T for $5 \mathrm{~min}$ each, the membranes were incubated with horseradish peroxidase-conjugated anti-rabbit $(1: 1,000 ; 7074$; Cell Signaling Technology, Inc.) or anti-mouse (1:1,000; 7076; Cell Signaling Technology, Inc.) secondary antibodies for $1 \mathrm{~h}$ at room temperature, and then washed three times with TBS-T. Blots were visualized and digitized using enhanced chemiluminescence (Thermo Fisher Scientific, Inc.) and the results were quantified with ImageJ v1.8.0 software (National Institutes of Health). The experiment was repeated three times.

Statistical analysis. All numerical data are presented as the mean \pm standard deviation. One-way analysis of variance with Bonferroni's method was performed with SPSS 24.0 software (IBM Corp.). $\mathrm{P}<0.05$ was considered to indicate a statistically significant difference.
Table I. Primers used in polymerase chain reaction.

\begin{tabular}{ll}
\hline Canine gene & \multicolumn{1}{c}{ Primers (5'-3') } \\
\hline BMP2 & F: CCCTACATGCTGGACCTGTA \\
Runx2 & F: ATTTCTGGCAGTTCTTCCAA \\
& R: GCGGAAGCATTCTGGAAGGAGAC \\
Col1 & F: GGTTCAGCTAAGTTGGAGGTACT \\
& R: CCAATGTTGCCAGGGTAAC \\
OCN & F: CTGGTCCAGCAGATGCAAAG \\
& R: CCGCTTGGACACGAAGGTT \\
BSP & F: TTGCTCAGCATTTTGGGAATGG \\
& R: AACGTGGCCGATACTTAAAGACC \\
ALP & F: TTCAAACCGAGACACAAGCACT \\
& R: GGGTCAGTCACGTTGTTCCTGT \\
DMP1 & F: CAGGAGCACAGGAAAAGGAG \\
& R: CTGGTGGTATCTTGGGCACT \\
DSPP & F: GTCCTAGTGGGAATGGAGCA \\
& R: TCTTCAGGGCCATCATCTTC \\
GAPDH & F: TGTCCCCACCCCCAATGTATC \\
& R: CTCCGATGCCTGCTTCACTACCTT
\end{tabular}

BMP2, bone morphogenetic protein 2; Runx2, Runt-related transcription factor 2; COL1, collagen 1; OCN, osteocalcin; BSP, bone sialoprotein; ALP, alkaline phosphatase; DMP1, dentin matrix protein-1; DSPP, dentin sialophosphoprotein; F, forward; R, reverse.

\section{Results}

Isolated cPDLSCs exhibit stem cell characteristics. cPDLSCs displayed a fibroblast-like or stellate-shaped morphology under an inverted microscope (Fig. 1A), while mesenchymal stem cell markers were examined by flow cytometric analysis (Fig. 1B). STRO-1, CD90, CD105, CD73 and CD45 were selected as the critical markers to compare the similarities between cPDLSCs and mesenchymal stem cells $(32,33)$. A relatively high number of cPDLSCs expressed CD73 (99.9\%), CD90 (99.9\%) and CD105 (99.9\%), while a low number of cPDLSCs expressed CD45 (2.04\%). STRO-1 was expressed at more moderate levels in cPDLSCs (28.2\%). Alizarin Red S and Oil Red $\mathrm{O}$ staining demonstrated the presence of mineralization (Fig. 1C) and lipid droplets (Fig. 1D) following induction in osteogenic and adipogenic media, respectively. In addition, neurogenic induction caused morphological changes and increased the expression of the neurogenic marker $\beta$ III-tubulin in cPDLSCs (Fig. 1E).

EphrinB2 gene-modified cPDLSCs exhibit increased GFP fluorescence, and $m R N A$ and protein expression levels of ephrinB2. The fluorescent-inverted phase-contrast microscope was used to detect the GFP expression in wild-type cPDLSCs, EfnB2-cPDLSCs and Vector-cPDLSCs. Using microscopy, GFP was detected in Vector-cPDLSCs and EfnB2-cPDLSCs, while no detectable fluorescence signal was observed in wild-type cPDLSCs (Fig. 2A). In addition, RT-qPCR and western blot analyses were performed to 

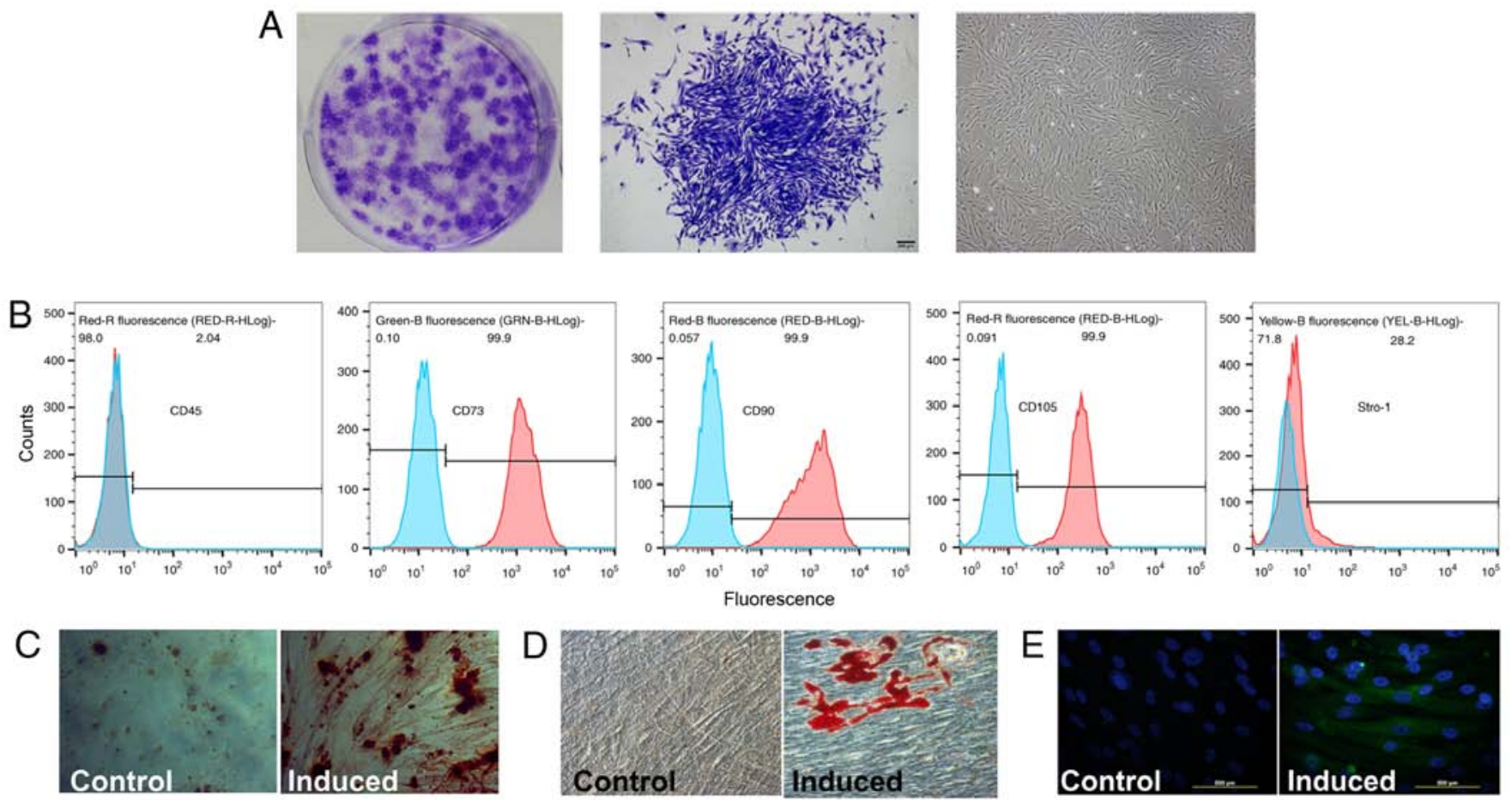

Figure 1. Characterization of cPDLSCs. (A) Colony formation and morphology of cPDLSCs. Magnification, $\mathrm{x} 4$. (B) Flow cytometric analysis of cell surface markers CD45, CD73, CD90, CD105 and STRO-1. Blue presents the blank control and red is the surface marker. (C) Alizarin Red S staining of mineralized nodule formation after 28 days of osteogenesis induction. Magnification, x20. (D) Oil Red O staining of lipid droplet after 28 days of adipogenesis induction. Magnification, x10. (E) Immunofluorescence imaging of $\beta$ III-tubulin after 28 days of neurogenesis induction (scale bar, $500 \mu \mathrm{m}$ ). cPDLSCs, canine periodontal ligament stem cells.

A

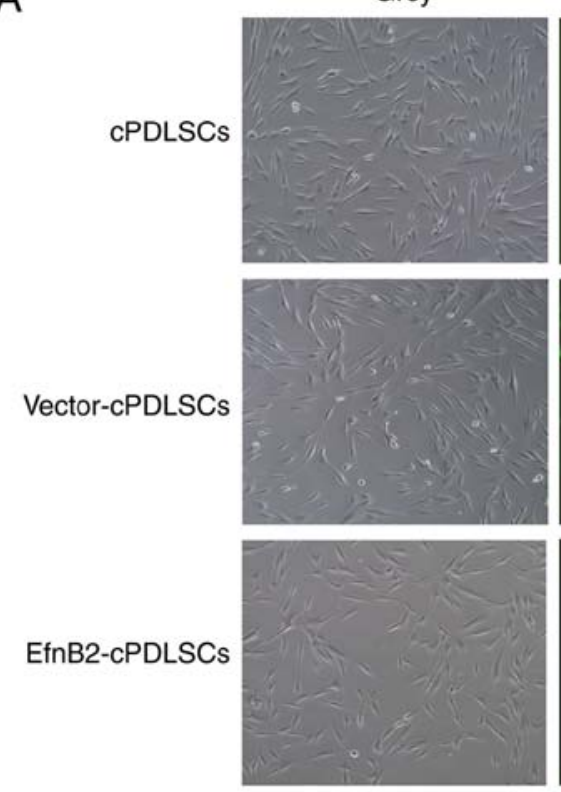

Influorescence
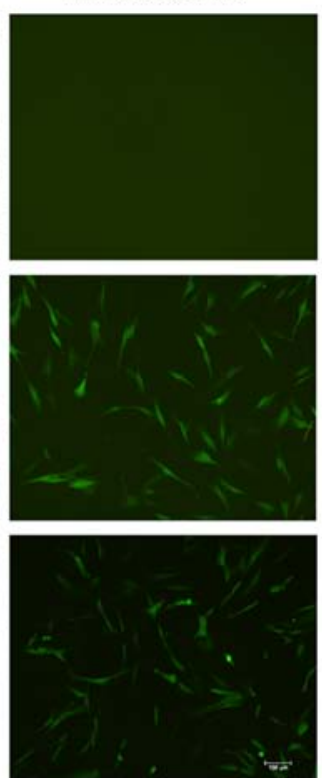

B

Relative mRNA expression
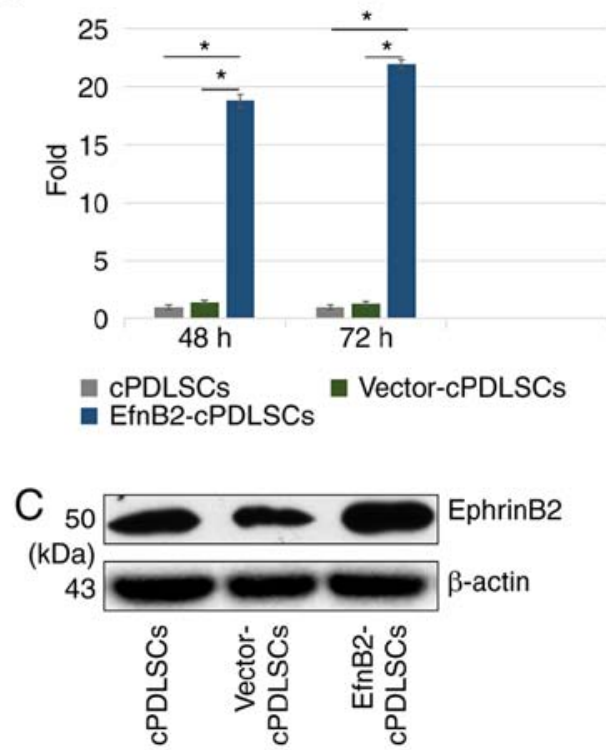

Figure 2. EphrinB2 gene transfection and identification of cPDLSCs. (A) Expression of green fluorescent protein in wild-type cPDLSCs, Vector-cPDLSCs and EfnB2-cPDLSCs. Magnification, x10. (B) Reverse transcription-quantitative polymerase chain reaction analysis of the ephrinB2 mRNA expression in wild-type cPDLSCs, Vector-cPDLSCs and EfnB2-cPDLSCs. (C) Western blot analysis of ephrinB2 protein expression in wild-type cPDLSCs, Vector-cPDLSCs and EfnB2-cPDLSCs. ${ }^{*} \mathrm{P}<0.05$. cPDLSCs, canine periodontal ligament stem cells.

respectively detect the mRNA and protein expression levels of ephrinB2 in wild-type cPDLSCs, EfnB2-cPDLSCs and Vector-cPDLSCs. It was observed that the expression of ephrinB2 mRNA was 20 -fold higher in EfnB2-cPDLSCs compared with that in cPDLSCs and Vector-cPDLSCs.
Western blot analysis confirmed the significantly increased protein expression levels of ephrinB2 in EfnB2-cPDLSCs compared with cPDLSCs and Vector-cPDLSCs. These results indicated the successful upregulation of EfnB2 gene in transfected cPDLSCs. 
A
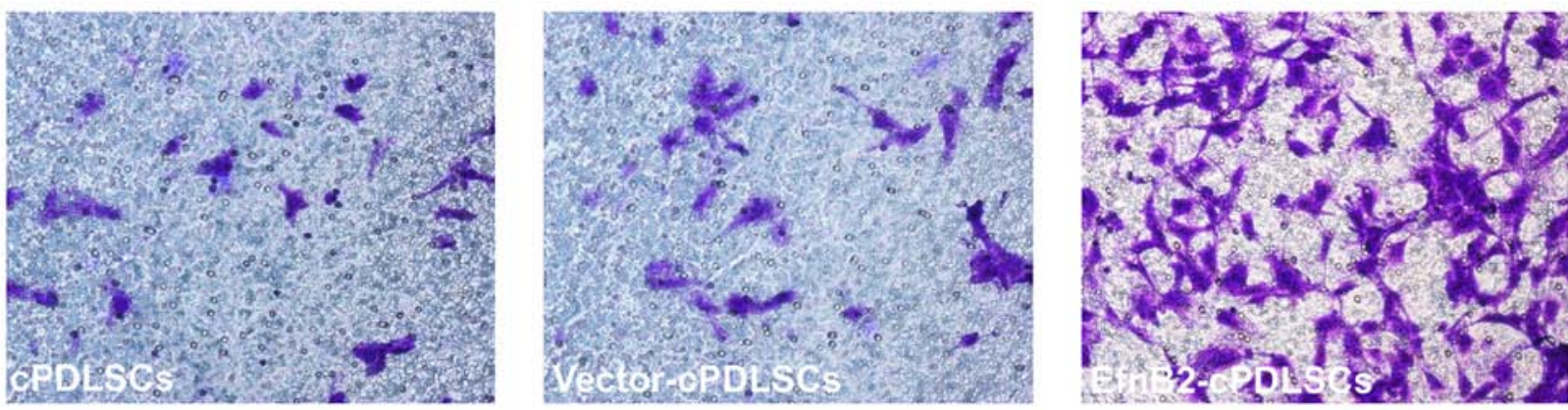

B

Cell migration

C

Cell proliferation
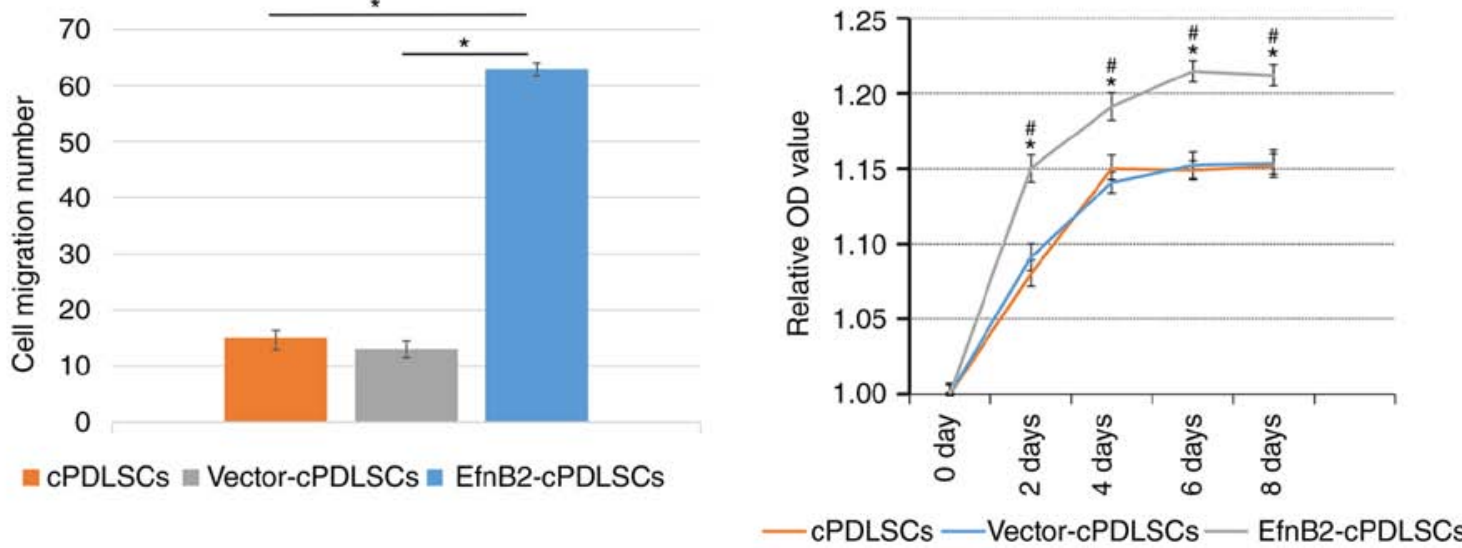

Figure 3. Cell migration and proliferation of cPDLSCs, Vector-cPDLSCs and EfnB2-cPDLSCs. (A) Images (magnification, x10) of cells that had migrated to the lower insert surface after $12 \mathrm{~h}$. (B) Relative number of migrated cPDLSCs, Vector-cPDLSCs and EfnB2-cPDLSCs. (C) Relative OD values of cPDLSCs, Vector-cPDLSCs and EfnB2-cPDLSCs on days 0,2, 4, 6 and 8 of the cell proliferation assay. Data are presented as the mean \pm standard deviation (n=6). ${ }^{*} \mathrm{P}<0.05$ vs. Vector-cPDLSCs; ${ }^{\#} \mathrm{P}<0.05$ vs. cPDLSCs. cPDLSCs, canine periodontal ligament stem cells; OD, optical density.

A

VectorcPDLSCs

EfnB2cPDLSCs

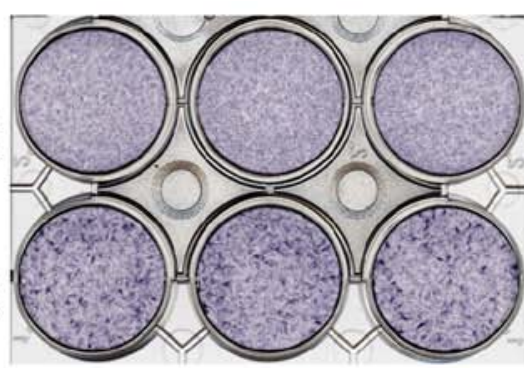

D

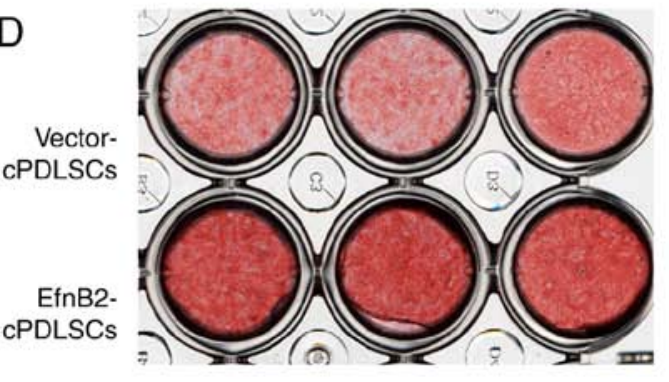

B

VectorcPDLSCs

EfnB2cPDLSCS

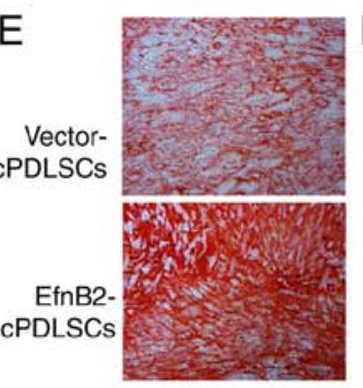

C Alkaline phosphatase assay after 14 days of osteogenic induction

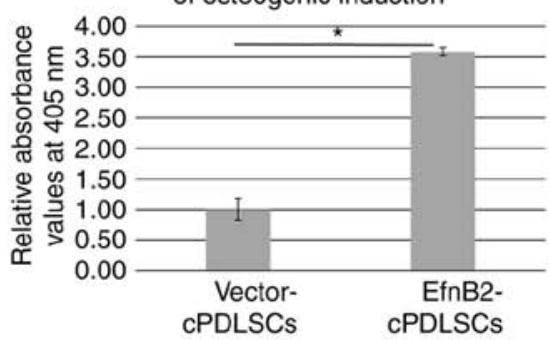

$\mathrm{F}$

Alizarin red staining after 21 days of osteogenic induction

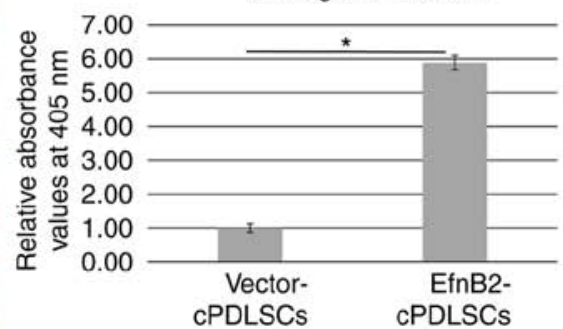

Figure 4. ALP and Alizarin Red S staining of transfected cells subjected to 14 and 21 days of osteogenic/odontogenic induction, respectively. (A) Gross appearance, and (B) light microscopy images (magnification, x10) of ALP-stained Vector-cPDLSCs and EfnB2-cPDLSCs after 14 days of osteogenic induction. (C) ALP activity in cell lysates. (D) Gross appearance, and (E) light microscopy images (magnification, x10) of Alizarin Red S-stained Vector-cPDLSCs and EfnB2-cPDLSCs after 21 days of osteogenic induction. (F) Quantification of Alizarin Red S staining intensity. *P<0.05. ALP, alkaline phosphatase; cPDLSCs, canine periodontal ligament stem cells.

EphrinB2 gene-modified cPDLSCs exhibit enhanced migration and proliferation. As observed in Fig. 3A,EfnB2-cPDLSCs exhibited a significantly increased migratory ability compared with the cPDLSCs and Vector-cPDLSCs $(\mathrm{P}<0.05)$. In addition, 
A
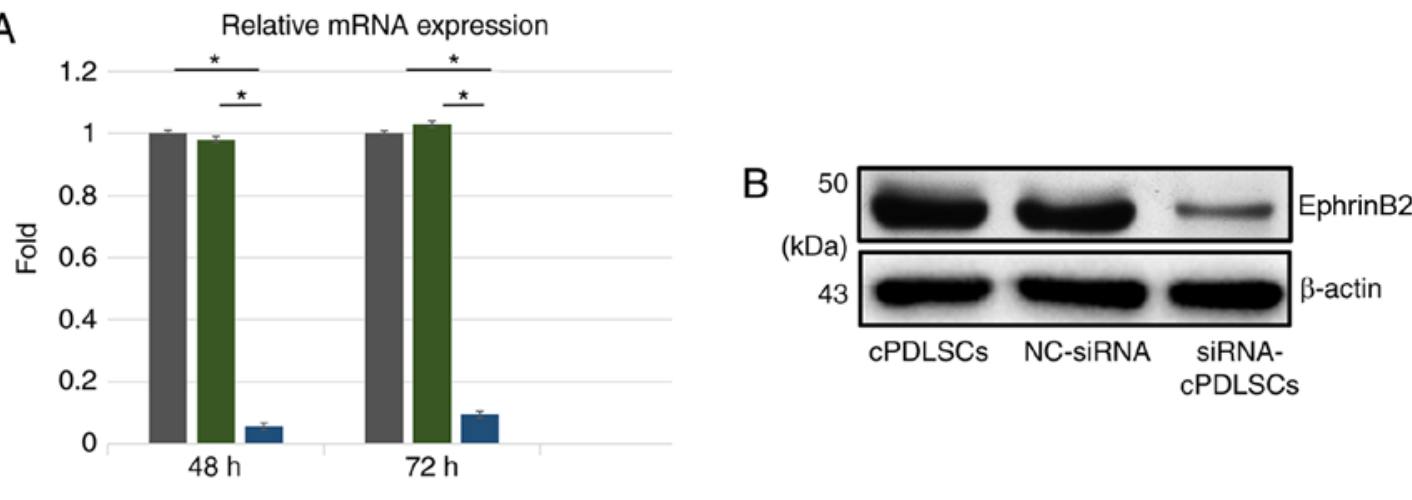

aPDLSCs

NC-siRNA

siRNA-cPDLSCs

Figure 5. EphrinB2 expression following transfection with EphrinB2-siRNA and FAM-labeled NC-siRNA. (A) mRNA expression levels of EphrinB2 in cPDLSCs following transfection for 48 and $72 \mathrm{~h}$. (B) Protein expression levels of EphrinB2 in cPDLSCs following transfection for $72 \mathrm{~h}$. "P<0.05. cPDLSCs, canine periodontal ligament stem cells; siRNA, small interfering RNA; NC, negative control.
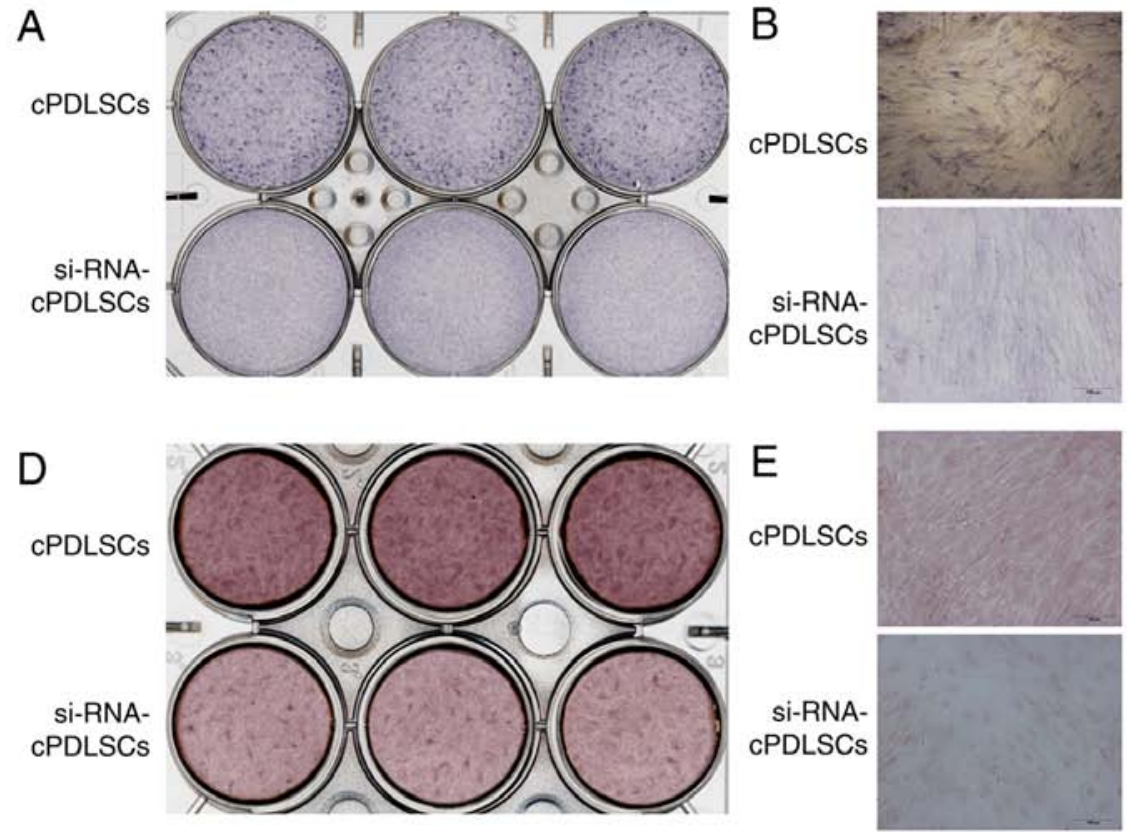

\section{C}

Alkaline phosphatase assay after 14 days of osteogenic induction
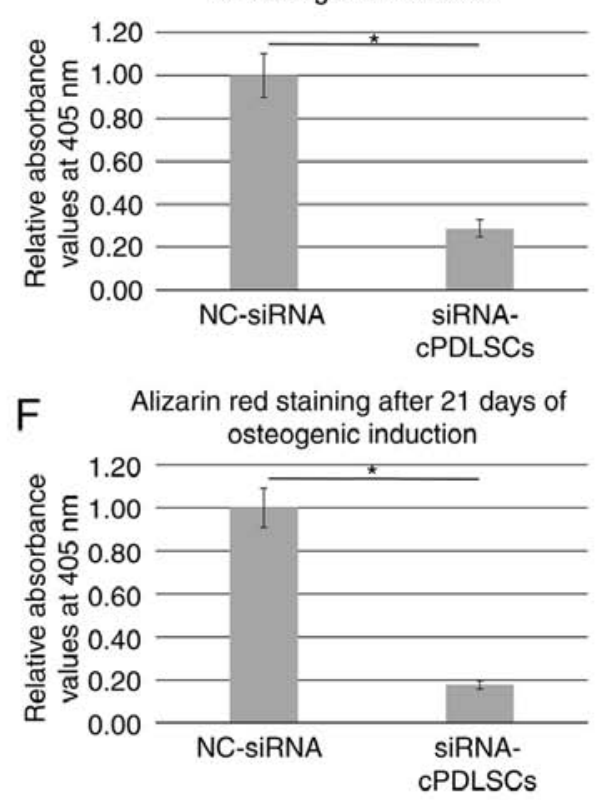

Figure 6. ALP and Alizarin Red S staining of siRNA-transfected cells subjected to 14 and 21 days of osteogenic/odontogenic induction, respectively. (A) Gross appearance, and (B) light microscopy images (magnification, x10) of ALP-stained cPDLSCs and siRNA-cPDLSCs following osteogenic induction for 14 days. (C) ALP activity in cell lysates. (D) Gross appearance, and (E) light microscopy images (magnification, x10) of Alizarin Red S stained cPDLSCs and siRNA-cPDLSCs following osteogenic induction for 21 days. (F) Quantification of Alizarin Red S staining intensity. "P<0.05. ALP, alkaline phosphatase; cPDLSCs, canine periodontal ligament stem cells.

EfnB2-cPDLSCs exhibited significantly enhanced proliferation on days 2, 4, 6 and 8 compared with the other two groups (Fig. 3B).

EphrinB2 gene-modified cPDLSCs exhibit enhanced osteogenic ability. To examine the osteogenic ability of cells, an ALP assay was performed following osteogenic induction for 14 days. As shown in Fig. 4A-C, EfnB2-cPDLSCs displayed an enhanced ALP activity compared with Vector-cPDLSCs. In addition, Alizarin Red S staining performed after 21 days of osteogenic induction revealed more prominent mineralized nodules in EfnB2-cPDLSCs compared with those observed in Vector-cPDLSCs (Fig. 4D-F).
Inhibition of EphrinB2 expression suppresses the osteogenic ability of cells. EphrinB2 expression was blocked with EphrinB2-siRNA transfection, as indicated by the significant reduction in the mRNA and protein levels of ephrinB2 in cPDLSCs (Fig. 5). Next, ALP staining was performed subsequent to osteogenic induction for 14 days. As shown in Fig. 6A-C, EphrinB2-siRNA-transfected cPDLSCs (siRNA-cPDLSCs) displayed decreased ALP activity as compared with the NC-siRNA-transfected cPDLSCs. In addition, Alizarin Red S staining performed after 21 days of osteogenic induction revealed a reduction in the mineralized nodules in siRNA-cPDLSCs as compared with those observed in cPDLSCs transfected with NC-siRNA (Fig. 6D-F). 
A

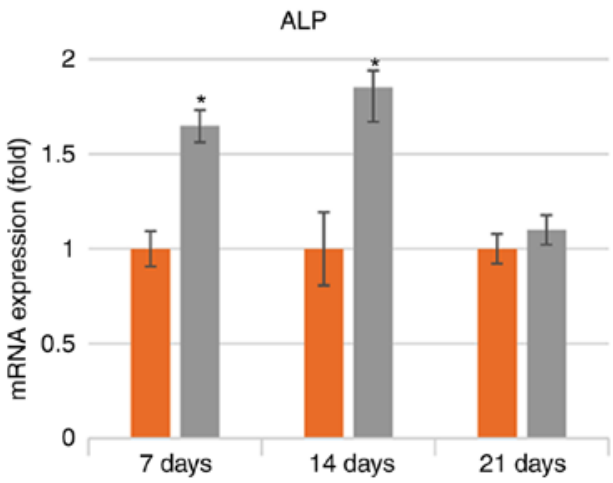

a Vector-CPDLSCs

C

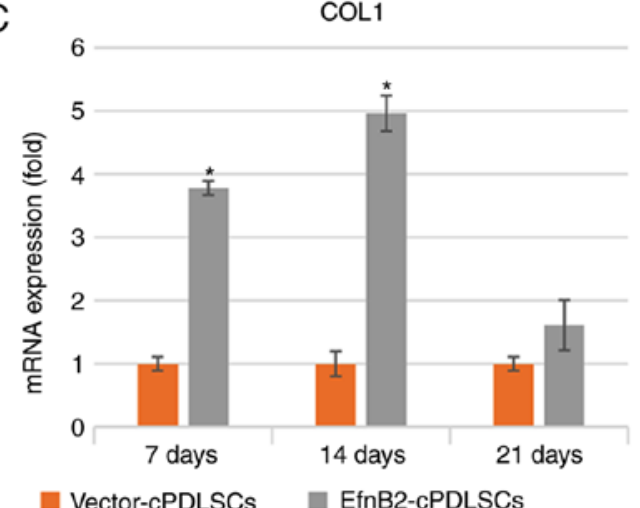

E

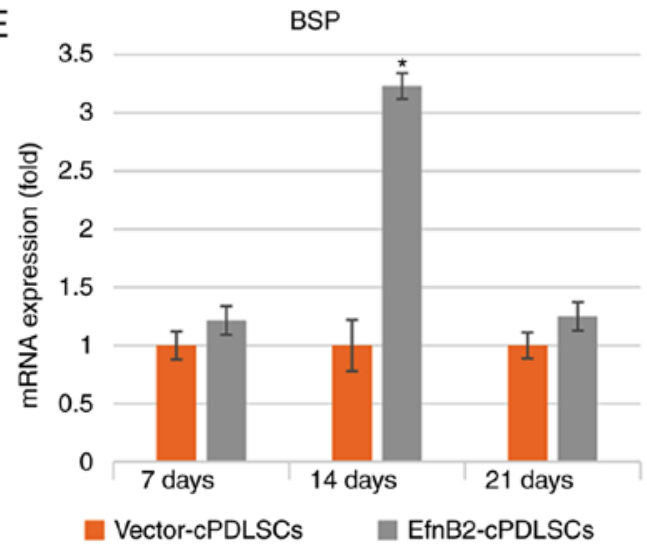

G

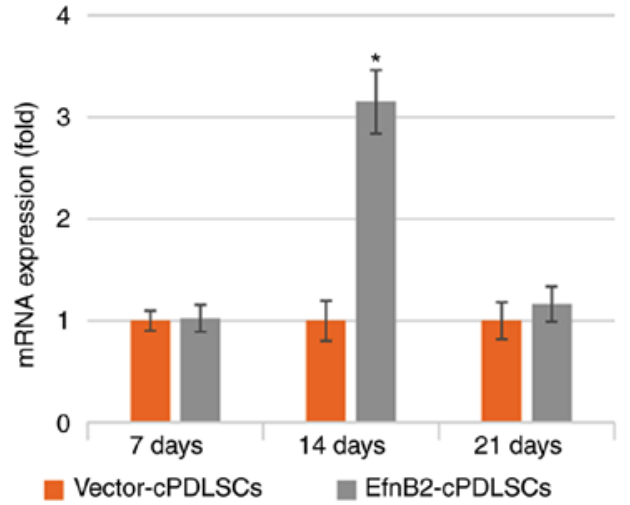

B

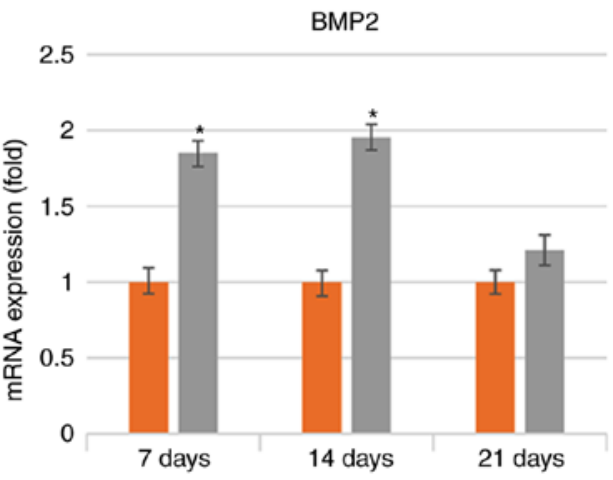

a Vector-cPDLSCs EfnB2-cPDLSCS

$\mathrm{D}$ RunX2

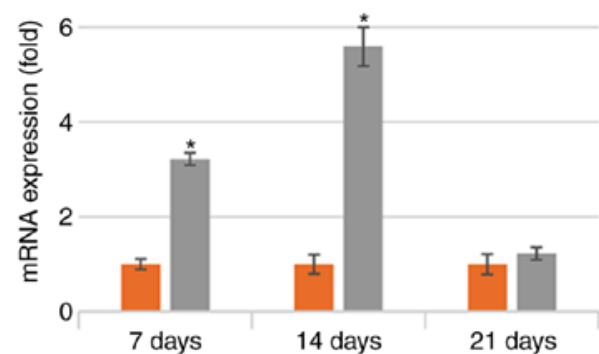

Vector-cPDLSCs EfnB2-cPDLSCs

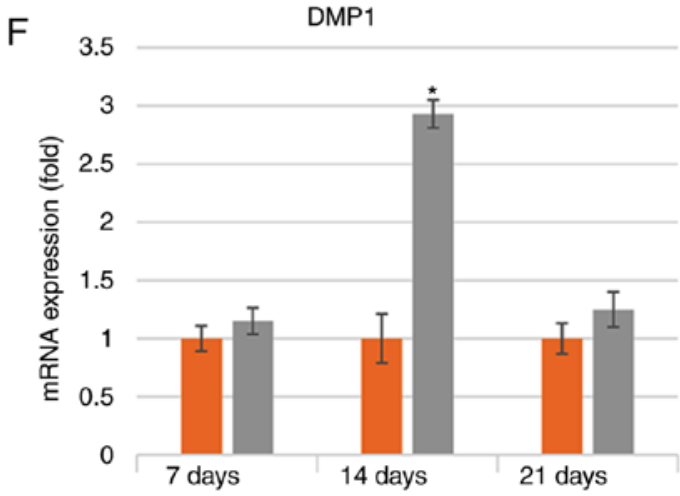

E Vector-cPDLSCs EfnB2-cPDLSCs

$\mathrm{H}$

OCN

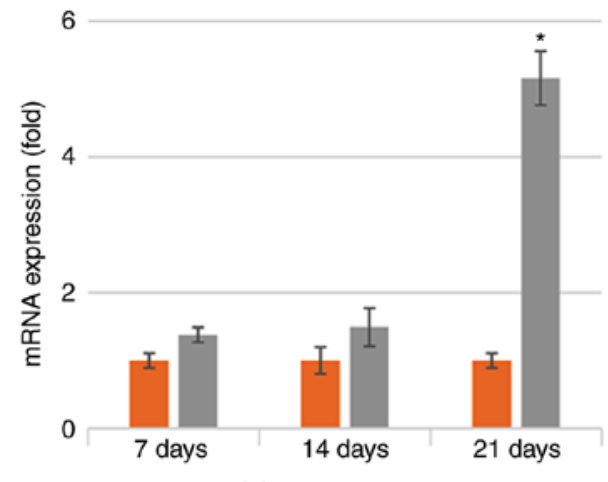

" Vector-cPDLSCs EfnB2-cPDLSCs

Figure 7. Reverse transcription-quantitative polymerase chain reaction analysis of mRNA expression levels of (A) ALP, (B) BMP2, (C) COL1, (D) Runx2, (E) BSP, (F) DMP1 (G) DSPP and (H) OCN in Vector-cPDLSCs and EfnB2-cPDLSCs after 7, 14 and 21 days of osteogenic/odontogenic induction. "P<0.05 vs. Vector-cPDLSCs. cPDLSCs, canine periodontal ligament stem cells; ALP, alkaline phosphatase; BMP2, bone morphogenetic protein 2; COL1, collagen 1; Runx2, Runt-related transcription factor 2; BSP, bone sialoprotein; DMP1, dentin matrix protein-1; DSPP, dentin sialophosphoprotein; OCN, osteocalcin. 
A
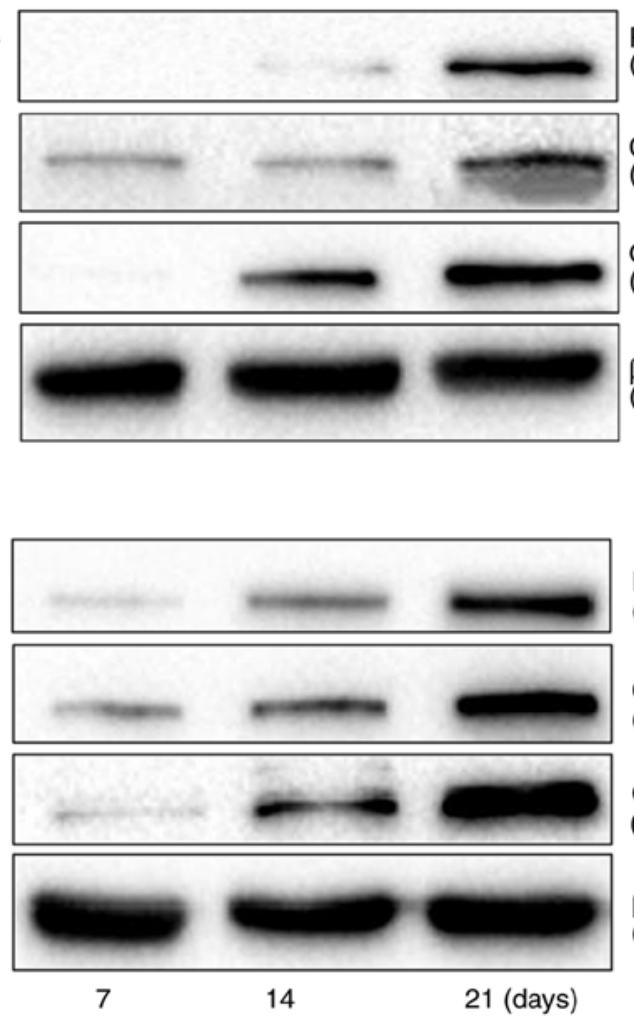

RunX2

(vector-cPDLSCs)

COL1

(vector-cPDLSCs)

$\mathrm{OCN}$

(vector-cPDLSCs)

$\beta$-actin

(vector-cPDLSCs)

RunX2

(EfnB2-cPDLSCs)

COL1

(EfnB2-cPDLSCs)

\section{OCN}

(EfnB2-cPDLSCs)

$\beta$-actin

(EfnB2-cPDLSCs)
B

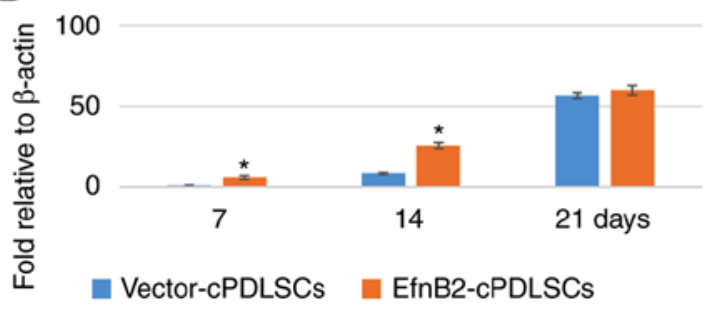

COL1

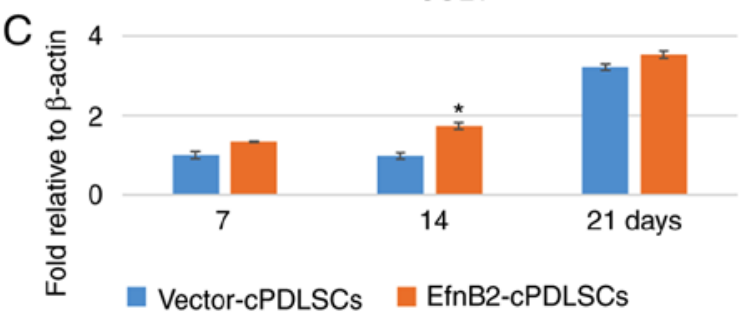

$\mathrm{D}$

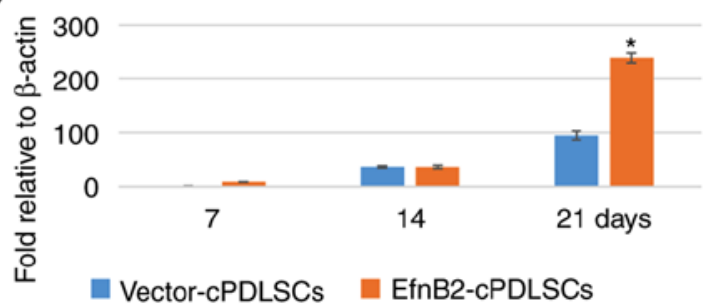

Figure 8. Endogenous protein expression of Runx2, COL1 and OCN during osteogenic differentiation of cPDLSCs. (A) Western blot analysis of protein expression levels of Runx2, COL1 and OCN in Vector-cPDLSCs and EfnB2-cPDLSCs after 7, 14 and 21 days of osteogenic/odontogenic induction. Quantification bands of (B) Runx2, (C) COL1 and (D) OCN in Vector-cPDLSCs and EfnB2-cPDLSCs. "P<0.05 vs. Vector-cPDLSCs. COL1, collagen 1; Runx2, Runt-related transcription factor 2; OCN, osteocalcin; cPDLSCs, canine periodontal ligament stem cells.

Effects of ephrinB2 transfection on the mRNA expression levels of osteogenic markers in cPDLSCs following osteogenic/odontogenic induction. As shown in Fig. 7, the mRNA expression levels of ALP, bone morphogenetic protein 2 (BMP2), COL1 and Runx2 were markedly increased in EfnB2-cPDLSCs compared with those in Vector-cPDLSCs on days 7 and 14 of osteogenic/odontogenic induction (Fig. 7A-D). However, no significant differences were observed on day 21. Furthermore, the mRNA expression levels of bone sialoprotein (BSP), dentin matrix protein-1 (DMP1) and dentin sialophosphoprotein (DSPP) were significantly higher in EfnB2-cPDLSCs after 14 days of induction, whereas no significant differences were observed on days 7 or 21 (Fig. 7E-G). For OCN mRNA expression, no significant differences were observed between EfnB2-cPDLSCs and Vector-cPDLSCs on days 7 and 14, whereas a significantly higher gene expression was observed on day 21 of osteogenic induction (Fig. 7H).

Protein expression levels of osteogenic markers in cPDLSCS following osteogenic/odontogenic induction. As shown in Fig. 8, the protein expression levels of Runx2 were increased in EfnB2-cPDLSCs compared with Vector-cPDLSCs on days 7 and 14 after osteogenic/odontogenic induction; however, no significant differences were observed on day 21 . In addition, the protein expression of OCN was significantly upregulated in EfnB2-cPDLSCs compared with Vector-cPDLSCs on day 21 , while no significant differences were observed on days 7 and 14. Notably, the protein expression levels of COL1 were increased in EfnB2-cPDLSCs compared with Vector-cPDLSCs on day 14; however, no significant difference were observed on days 7 and 21.

Endogenous levels of phosphorylated and unphosphorylated ephrinB2 and EphB4 proteins during osteogenic/odontogenic differentiation of EfnB2-cPDLSCs and Vector-cPDLSCs. The western blot results revealed that maximal levels of phospho-ephrinB2 occurred $30 \mathrm{~min}$ after stimulation of EfnB2-cPDLSCs and Vector-cPDLSCs with EphB4-FC (Fig. 9A and B). The protein expression levels of phospho-ephrinB2 remained increased in EfnB2-cPDLSCs after $12 \mathrm{~h}$ of osteogenic/odontogenic induction and reached a maximum at $24 \mathrm{~h}$ (Fig. 9C-E). Although phospho-ephrinB2 was detected at a low expression level in Vector-cPDLSCs at $12 \mathrm{~h}$, and reached a maximum at $24 \mathrm{~h}$, the expression levels were markedly lower compared with EfnB2-cPDLSCs. Similarly, there was a significant increase of phospho-EphB4 in EfnB2-cPDLSCs compared with Vector-cPDLSCs at $48 \mathrm{~h}$ (Fig. 9C-E).

\section{Discussion}

Currently, the selection of a carrier scaffold, signaling molecules and seed cells is one of the key issues in periodontal tissue engineering. A previous study has reported 
A
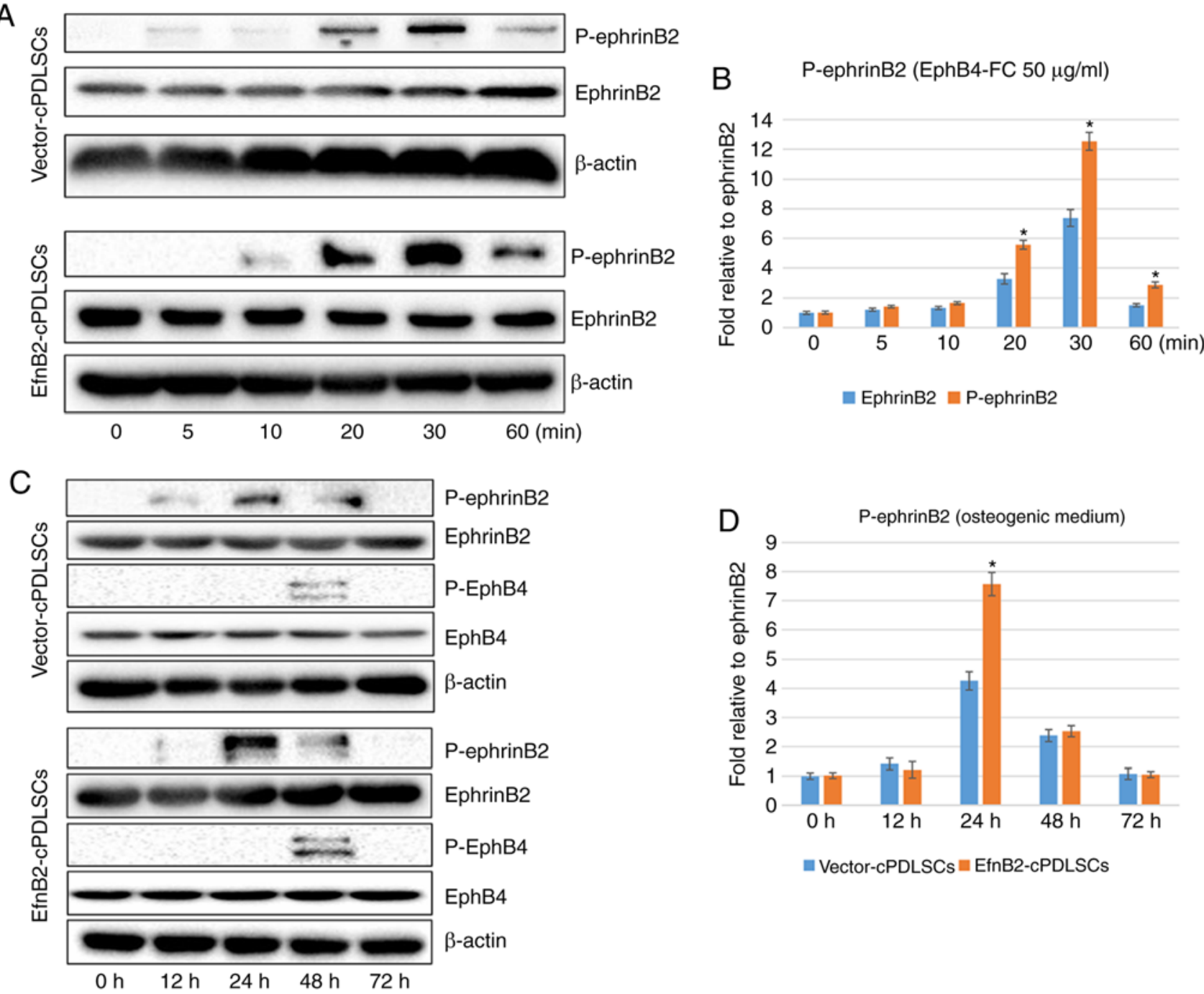

EphrinB2 $\mathbf{E}$ P-ephrinB2

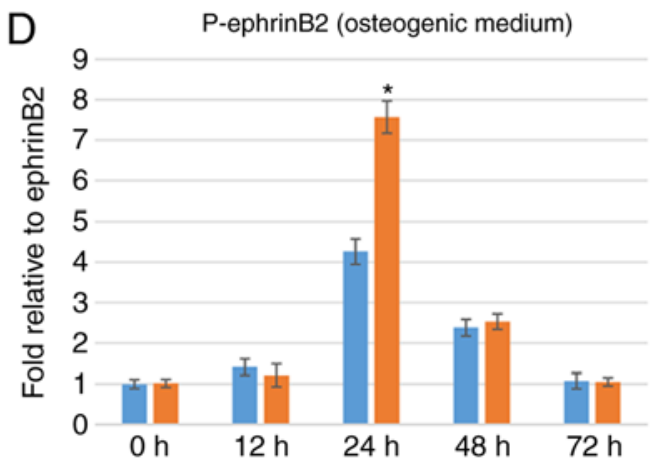

= Vector-cPDLSCs $=$ EfnB2-cPDLSCs

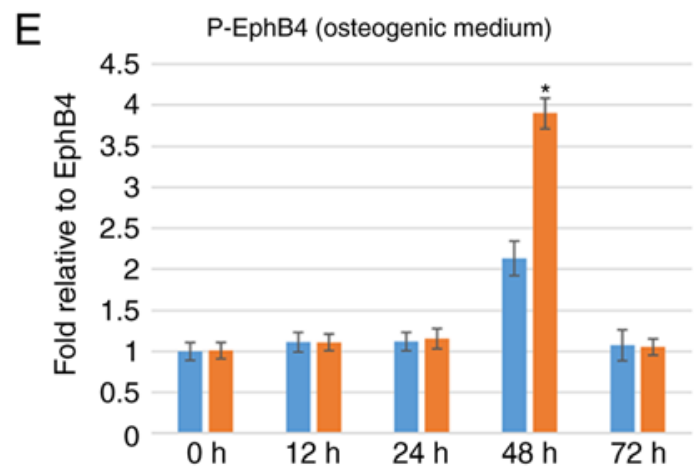

= Vector-cPDLSCs $\equiv$ EfnB2-cPDLSCs

Figure 9. Endogenous expression of EphrinB2, EphB4 and their phosphorylated proteins during osteogenic differentiation of cPDLSCs. (A) Western blots of EphrinB2 and P-ephrinB2 in Vector-cPDLSCs and EfnB2-cPDLSCs stimulated with EphB4-FC for 0, 5, 10, 20,30 and 60 min. Quantification of protein expression bands of (B) P-ephrinB2 stimulated with EphB4-FC for 0, 5, 10, 20, 30 and 60 min. (C) Western blots of EphrinB2, P-ephrinB2, EphB4 and P-EphB4 in Vector-cPDLSCs and EfnB2-cPDLSCs following osteogenic induction for 0, 12, 24, 48 and $72 \mathrm{~h}$. Quantification of protein expression bands of (D) P-ephrinB2 and (E) P-EphB4 following osteogenic induction for 0, 12, 24, 48 and $72 \mathrm{~h}$. ${ }^{*} \mathrm{P}<0.05$ vs. Vector-cPDLSCs. cPDLSCs, canine periodontal ligament stem cells; P-, phosphorylated.

that ephrinB2 ligand and its receptor EphB4 constitute a bidirectional signaling pathway with a crucial role in bone remodeling (8). It has been indicated that the ephrinB2-mediated reverse signaling pathway inhibits osteoclast function and bone resorption by reducing the activity of c-Fos and NFATc1, whereas the forward signaling pathway promotes bone formation by reducing RhoA activity and enhancing osteoblast differentiation (9).

Numerous studies have attempted to use ephrinB2 as a molecular therapy for tissue engineering. Indeed, in animal 
experiments, it has been demonstrated that the degree of bone mineralization, bone hardness and osteoblast differentiation in ephrinB2-deficient mice was significantly reduced compared with those in wild-type mice (34). Our previous study revealed that that ALP activity of PDLSCs was significantly increased following transfection with ephrinB2 in vitro, which resulted in more calcified nodules and increased expression levels of osteogenesis-associated genes (BSP, COL1, Runx2, DSPP and OCN), suggesting that upregulation of ephrinB2 promoted the osteogenic differentiation of PDLSCs (13). Collectively, these previous results suggested that ephrinB2 is a potentially useful biomolecule for tissue engineering. However, although in vitro and in vivo studies have been performed, the effect of bidirectional ephrinB2-EphB4 signaling has not been examined in cPDLSCs, and thus this was the focus of the present study.

The present study first examined the stem cell-like characteristics of isolated cPDLSCs, including self-renewal capacity, multilineage differentiation ability and expression levels of stem cell surface markers. It was observed that isolated cPDLSCs displayed a fibroblast-like or stellate-shaped morphology, and grew in colonies. A characteristic set of mesenchymal stem cells markers, including STRO-1, CD90, CD105, CD73 and CD45, were selected to perform a flow cytometric assay $(32,33)$. A percentage of cPDLSCs as high as $28.2 \%$ expressed STRO-1, which is considered to be a mesenchymal stem cell surface marker (35). In addition, Alizarin Red S staining for mineralized nodules, Oil Red O staining for lipid droplets and immunofluorescence staining for $\beta$ III-tubulin were also performed following induction of osteogenesis, adipogenesis or neurogenesis using the appropriate media. The findings suggested that cPDLSCs may be a favorable candidate for dental tissue engineering.

Next, transfection of cPDLSCs with ephrinB2 was conducted in the present study, and the fluorescent protein expression, as well as the mRNA and protein expression levels of ephrinB2 were examined. The results demonstrated continuous and stable expression of ephrinB2 mRNA and protein in EfnB2-cPDLSCs, thus verifying the successful transfection of ephrinB2 into cPDLSCs. In addition, to evaluate the proliferative and migratory abilities of cells following ephrinB2 transfection, Transwell and CCK-8 assays were performed, as previously described (13). EfnB2-cPDLSCs exhibited enhanced migration and proliferation compared with cPDLSCs and Vector-cPDLSCs. These results are consistent with previous research demonstrating that ephrinB2 reverse signaling promotes cell migration (36) and mediates cell proliferation (37).

To further investigate the effect of ephrinB2 gene transfection on osteogenic differentiation of cPDLSCs, ALP and Alizarin Red S staining assays were also performed in the current study. As expected, when cells were cultured under osteoinductive conditions, EfnB2-cPDLSCs displayed enhanced ALP activity and formed more mineralized nodules as compared with Vector-cPDLSCs. In addition, the expression of middle and late osteogenic genes and proteins was significantly increased, suggesting that ephrinB2 signaling was associated with late bone metabolism (38). Notably, in contrast to our previous study (39), in which EphB4-FC was used to stimulate human PDLSCs and no significant differences were observed in the expression levels of early osteogenic genes in the EphB4-FC-stimulated groups, there were significant differences in the gene expression levels of early osteogenic genes in EfnB2-cPDLSCs in the present study. Thus, it is speculated that ephrinB2 reverse signaling is associated not only with bone metabolism, but also with early bone formation (34). Furthermore, EphB4-FC stimulation can only establish ephemeral and unsustainable signaling, while gene transfection of ephrinB2 is more intense and sustainable (40).

Previous research reported that activation of the ephrinB2-EphB4 signaling pathway promoted early osteogenic differentiation, leading to earlier osteogenic process and earlier expression of Runx2 (41); However, other research reported that high levels of ephrinB2 overexpression increases the osteogenic differentiation of human mesenchymal stem cells, while no significant changes in Runx 2 expression were found when they attempted to elucidate the molecular mechanisms of ephrinB2 overexpression (42). This suggests that the anaphase effect of cell-mediated mineralization is associated with other signaling pathways.

To further evaluate the effect and mechanisms of ephrinB2 and its receptor EphB4, western blot assays were conducted in the present study. Following stimulation with EphB4-FC, phospho-ephrinB2 expression was detectable. Notably, the phospho-ephrinB2 proteinexpression levelsinEfnB2-cPDLSCs were significantly higher than that in Vector-cPDLSCs. Following osteogenic/odontogenic induction, expression of phospho-ephrinB2 and phospho-EphB4 proteins was detected in EfnB2-cPDLSCs and Vector-cPDLSCs, demonstrating that reverse and forward signaling was induced. These results are consistent with the findings of previous studies, suggesting that ephrinB2 signaling can be activated, and that forward and reverse signaling can be induced in cPDLSCs $(43,44)$.

In conclusion, the findings of the present study indicated that ephrinB2 gene-modified cPDLSCs exhibited enhanced osteogenic differentiation, and that the ephrinB2 reverse signaling and EphB4 forward signaling pathways served a key role in this process. In addition, ephrinB2 gene modification promoted cell migration and proliferation. Based on these results, it is speculated that, since ephrinB2 is a transmembrane protein, it can only transmit signals through direct contact between cells; thus, when it is used for endogenous periodontal tissue regeneration, ephrinB2-expressing cells can only selectively come into direct contact with EphB4-expressing cells. Although this limits the therapeutic effect to the defect area, as a result, there are no side effects caused by diffusion of secretory cytokines following transplantation. Furthermore, it is proposed that the migratory ability of endogenous stem cells following transfection is enhanced, and thus these cells become more conducive to migration toward the tissue defect area, which is a key factor for tissue repair.

\section{Acknowledgements}

Not applicable.

\section{Funding}

This study was supported by the Seed Funding of Xuzhou Medical University (grant no. 2018KJ22), the Xuzhou Science 
and Technology Project (grant no. KC19137) and the National Natural Science Foundation Youth Science Foundation Project (grant no. 81700954).

\section{Availability of data and materials}

The datasets used and/or analyzed during the current study are available from the corresponding author on reasonable request.

\section{Authors' contributions}

PW and MG conceived and designed the study. SZ, ZL and CY performed the experiments. YL, YY and HW analyzed the data. SZ and CZ were responsible for interpreting the data and writing the manuscript. All authors read and approved the final manuscript.

\section{Ethics approval and consent to participate}

Ethical approval for the present study was obtained from the Institutional Animal Care and Use Committee of Xuzhou Medical University (Xuzhou, China).

\section{Patient consent for publication}

Not applicable.

\section{Competing interests}

The authors declare that they have no competing interests.

\section{References}

1. Pihlstrom BL, Michalowicz BS and Johnson NW: Periodontal diseases. Lancet 366: 1809-1820, 2005.

2. Taba M Jr, Kinney J, Kim AS and Giannobile WV: Diagnostic biomarkers for oral and periodontal diseases. Dent Clin North Am 49: 551-571, vi, 2005.

3. Darveau RP: Periodontitis: A polymicrobial disruption of host homeostasis. Nat Rev Microbiol 8: 481-490, 2010.

4. Heitz-Mayfield LJ and Lang NP: Surgical and nonsurgical periodontal therapy. Learned and unlearned concepts. Periodontol 2000 62: 218-231, 2013.

5. Pellegrini G, Pagni G and Rasperini G: Surgical approaches based on biological objectives: GTR versus GBR techniques. Int J Dent 2013: 521547, 2013.

6. Xu X, Li X, Wang J, He XT, Sun HH and Chen FM: Concise review: Periodontal tissue regeneration using stem cells: Strategies and translational considerations. Stem Cells Trans Med 8: 392-403, 2019.

7. Venkataiah VS, Handa K, Njuguna MM, Hasegawa T, Maruyama K, Nemoto E, Yamada S, Sugawara S, Lu L, Takedachi M, et al: Periodontal regeneration by allogeneic transplantation of adipose tissue derived multi-lineage progenitor stem cells in vivo. Sci Rep 9: 921, 2019.

8. Zhao C, Irie N, Takada Y, Shimoda K, Miyamoto T, Nishiwaki T, Suda T and Matsuo K: Bidirectional ephrinB2-EphB4 signaling controls bone homeostasis. Cell Metab 4: 111-121, 2006.

9. Pasquale EB: Eph-ephrin bidirectional signaling in physiology and disease. Cell 133: 38-52, 2008.

10. Bochenek ML, Dickinson S, Astin JW, Adams RH and Nobes CD: Ephrin-B2 regulates endothelial cell morphology and motility independently of Eph-receptor binding. J Cell Sci 123: 1235-1246, 2010.

11. Pennisi A, Ling W, Li X, Khan S, Shaughnessy JD Jr, Barlogie B and Yaccoby S: The ephrinB2/EphB4 axis is dysregulated in osteoprogenitors from myeloma patients and its activation affects myeloma bone disease and tumor growth. Blood 114: 1803-1812, 2009.
12. Füller T, Korff T, Kilian A, Dandekar G and Augustin HG: Forward EphB4 signaling in endothelial cells controls cellular repulsion and segregation from ephrinB2 positive cells. J Cell Sci 116: 2461-2470, 2003.

13. Zhu SY, Wang PL, Liao CS, Yang YQ, Yuan CY, Wang S, Dissanayaka WL, Heng BC and Zhang CF: Transgenic expression of ephrinB2 in periodontal ligament stem cells (PDLSCs) modulates osteogenic differentiation via signaling crosstalk between ephrinB2 and EphB4 in PDLSCs and between PDLSCs and pre-osteoblasts within co-culture. J Periodontal Res 52: 562-573, 2017.

14. Yuan C, Wang P, Zhu S, Zou T, Wang S, Xu J, Heng BC, Diogenes A and Zhang C: EphrinB2 stabilizes vascularlike structures generated by endothelial cells and stem cells from apical papilla. J Endod 42: 1362-1370, 2016.

15. Gong T, Heng BC, Xu J, Zhu S, Yuan C, Lo EC and Zhang C: Decellularized extracellular matrix of human umbilical vein endothelial cells promotes endothelial differentiation of stem cells from exfoliated deciduous teeth. J Biomed Mater Res A 105: 1083-1093, 2017.

16. Gronthos S, Mankani M, Brahim J, Robey PG and Shi S: Postnatal human dental pulp stem cells (DPSCs) in vitro and in vivo. Proc Natl Acad Sci USA 97: 13625-13630, 2000.

17. Miura M, Gronthos S, Zhao M, Lu B, Fisher LW, Robey PG and Shi S: SHED: Stem cells from human exfoliated deciduous teeth. Proc Natl Acad Sci USA 100: 5807-5812, 2003.

18. Huang GT, Sonoyama W, Liu Y, Liu H, Wang S and Shi S: The hidden treasure in apical papilla: The potential role in pulp/dentin regeneration and bioroot engineering. J Endod 34: 645-651, 2008.

19. Zhang Z, Nor F, Oh M, Cucco C, Shi S and Nör JE: Wnt/ $\beta$-catenin signaling determines the vasculogenic fate of postnatal mesenchymal stem cells. Stem Cells 34: 1576-1587, 2016.

20. Bento LW, Zhang Z, Imai A, Nör F, Dong Z, Shi S, Araujo FB and Nör JE: Endothelial differentiation of SHED requires MEK1/ERK signaling. J Dent Res 92: 51-57, 2013.

21. Dissanayaka WL, Zhu L, Hargreaves KM, Jin L and Zhang C: Scaffold-free prevascularized microtissue spheroids for pulp regeneration. J Dent Res 93: 1296-1303, 2014.

22. Cordeiro MM, Dong Z, Kaneko T, Zhang Z, Miyazawa M, Shi S, Smith AJ and Nör JE: Dental pulp tissue engineering with stem cells from exfoliated deciduous teeth. J Endod 34: 962-969, 2008.

23. Zhang N, Chen B, Wang W, Chen C, Kang J, Deng SQ, Zhang B, Liu S and Han F: Isolation, characterization and multi-lineage differentiation of stem cells from human exfoliated deciduous teeth. Mol Med Rep 14: 95-102, 2016.

24. Qazi TH, Mooney DJ, Pumberger M, Geissler S and Duda GN: Biomaterials based strategies for skeletal muscle tissue engineering: Existing technologies and future trends. Biomaterials 53: 502-521, 2015.

25. Akizuki T, Oda S, Komaki M, Tsuchioka H, Kawakatsu N, Kikuchi A, Yamato M, Okano T and Ishikawa I: Application of periodontal ligament cell sheet for periodontal regeneration: A pilot study in beagle dogs. J Periodontal Res 40: 245-251, 2005.

26. Iwata $T$, Yamato $M$, Tsuchioka H, Takagi R, Mukobata S, Washio K, Okano T and Ishikawa I: Periodontal regeneration with multi-layered periodontal ligament-derived cell sheets in a canine model. Biomaterials 30: 2716-2723, 2009.

27. Tsumanuma Y, Iwata T, Washio K, Yoshida T, Yamada A, Takagi R, Ohno T,Lin K, Yamato M,Ishikawa I, et al: Comparison of different tissue-derived stem cell sheets for periodontal regeneration in a canine 1-wall defect model. Biomaterials 32: 5819-5825, 2011.

28. Bugueño J, Li W, Salat P, Qin L and Akintoye SO: The bone regenerative capacity of canine mesenchymal stem cells is regulated by site-specific multilineage differentiation. Oral Surg Oral Med Oral Pathol Oral Radiol 123: 163-172, 2017.

29. Dissanayaka WL, Zhu X, Zhang C and Jin L: Characterization of dental pulp stem cells isolated from canine premolars. J Endod 37: 1074-1080, 2011.

30. Albini A and Benelli R: The chemoinvasion assay: A method to assess tumor and endothelial cell invasion and its modulation. Nat Protoc 2: 504-511, 2007.

31. Livak KJ and Schmittgen TD: Analysis of relative gene expression data using real-time quantitative PCR and the 2(-Delta Delta C(T)) method. Methods 25: 402-408, 2001.

32. Hermida-Gómez T, Fuentes-Boquete I, Gimeno-Longas MJ, Muiños-López E, Díaz-Prado S, de Toro FJ and Blanco FJ: Quantification of cells expressing mesenchymal stem cell markers in healthy and osteoarthritic synovial membranes. J Rheumatol 38: 339-349, 2011 
33. Greco SJ, Liu K and Rameshwar P: Functional similarities among genes regulated by OCT4 in human mesenchymal and embryonic stem cells. Stem Cells 25: 3143-3154, 2007.

34. Tonna S, Takyar FM, Vrahnas C, Crimeen-Irwin B, Ho PW, Poulton IJ, Brennan HJ, McGregor NE, Allan EH, Nguyen $\mathrm{H}$, et al: EphrinB2 signaling in osteoblasts promotes bone mineralization by preventing apoptosis. FASEB J 28: 4482-4496, 2014.

35. Ning H, Lin G, Lue TF and Lin CS: Mesenchymal stem cell marker Stro-1 is a $75 \mathrm{kd}$ endothelial antigen. Biochem Biophys Res Commun 413: 353-357, 2011.

36. Nakada M, Anderson EM, Demuth T, Nakada S, Reavie LB, Drake KL, Hoelzinger DB and Berens ME: The phosphorylation of ephrin-B2 ligand promotes glioma cell migration and invasion. Int J Cancer 126: 1155-1165, 2010.

37. Steinle JJ, Meininger CJ, Chowdhury U, Wu G and Granger HJ: Role of ephrin B2 in human retinal endothelial cell proliferation and migration. Cell Signal 15: 1011-1017, 2003.

38. Edwards CM and Mundy GR: Eph receptors and ephrin signaling pathways: A role in bone homeostasis. Int J Med Sci 5: 263-272, 2008.

39. Heng BC, Wang S, Gong T, Xu J, Yuan C and Zhang C: EphrinB2 signaling enhances osteogenic/odontogenic differentiation of human dental pulp stem cells. Arch Oral Biol 87: 62-71, 2018.

40. Ikeda Y, Sun Z, Ru X, Vandenberghe LH and Humphreys BD: Efficient gene transfer to kidney mesenchymal cells using a synthetic adeno-associated viral Vector. J Am Soc Nephrol 29: 2287-2297, 2018
41. Wang L, Zhang J, Wang C, Qi Y, Du M, Liu W, Yang C and Yang P: Low concentrations of TNF- $\alpha$ promote osteogenic differentiation via activation of the ephrinB2-EphB4 signalling pathway. Cell Prolif 50, 2017.

42. Tierney EG, Mcsorley K, Hastings CL, Cryan SA, O'Brien T, Murphy MJ, Barry FP, O'Brien FJ and Duffy GP: High levels of ephrinB2 over-expression increases the osteogenic differentiation of human mesenchymal stem cells and promotes enhanced cell mediated mineralisation in a polyethyleneimine-ephrinB2 gene-activated matrix. J Control Release 165: 173-182, 2013.

43. Li C, Shi C, Kim J, Chen Y, Ni S, Jiang L, Zheng C, Li D, Hou J, Taichman RS and Sun H: Erythropoietin promotes bone formation through EphrinB2/EphB4 signaling. J Dent Res 94: 455-463, 2015.

44. Matsuo K and Otaki N: Bone cell interactions through Eph/ephrin: Bone modeling, remodeling and associated diseases. Cell Adh Migr 6: 148-156, 2012.

This work is licensed under a Creative Commons Attribution-NonCommercial-NoDerivatives 4.0 International (CC BY-NC-ND 4.0) License. 